Potential Crash Risks of Expressway On-ramps and Off-ramps: A Case Study in Beijing, China

\author{
Xiaobo Qu ${ }^{a l}$, Ying Yang ${ }^{a}$, Zhiyuan Liu $^{b}$, Sheng Jin ${ }^{c}$, and Jinxian Weng ${ }^{d}$ \\ ${ }^{a}$ Griffith School of Engineering, Griffith University, Gold Coast QLD 4222, Australia \\ ${ }^{b}$ School of Civil Engineering, Monash University, Clayton VIC 3800, Australia \\ ${ }^{c}$ College of Civil Engineering and Architecture, Zhejiang University, Hangzhou 310058 \\ China \\ ${ }^{d}$ MOE Key Laboratory for Urban Transportation Complex Systems Theory and Technology, \\ Beijing Jiaotong University, 100044,China
}

\footnotetext{
${ }^{1}$ Corresponding author: Dr. Xiaobo Qu, x.qu@griffith.edu.au; Tel.: +61-7-55529724; Fax +61-7-55528065.
} 


\title{
Potential Crash Risks of Expressway On-ramps and Off-ramps: A Case Study in Beijing, China
}

\begin{abstract}
Objective: This paper aims to assess the potential crash risks across different traffic lanes (shoulder lane, median lane, and middle lane) near to ramps (before on-ramps, between ramps, and after off-ramps).

Methods: Field data are collected at seven locations (90 minutes for each location) during peak hours. Two risk indices based on time to collision, individual risk and societal risk are proposed to represent the distinct interpretations of risks to transport agencies and individual motorists. One-way ANOVA is applied to analyse the risk impact of ramps in various types of locations across distinct traffic lanes.

Results: Median lanes and sections after off-ramps have relatively lower risks compared to other lanes and sections. The individual and societal risks might not always be consistent since the two risk indices are proposed based on distinct perspectives: transport agencies focus on the crash/conflict frequencies in a road section and individual motorists are more concerned about the probability of being involved in a crash/conflict during their journey.

Conclusions: The differences in risks mainly result from the frequent lane-changing and merging activities. This model could be used to evaluate the performance of expressway ramp design.
\end{abstract}

Key words: Crash risk; On-ramps and off-ramps; Urban expressways 


\section{INTRODUCTION}

Urban expressways are important infrastructures serving intra-city transport needs. Vehicle crashes on expressways are one of the world's largest public health problems, especially in the sense that the victims are overwhelmingly healthy prior to the crashes (Pei et al., 2012; $\mathrm{Qu}$ et al., 2014). Indeed, road accidents are the leading cause of deaths for those under 45 years old (BITRE, 2006). Traffic safety issues are the most severe in developing countries, and simple prevention measures could halve the number of deaths. In this regard, traffic safety management has become an over-riding issue in developing countries, especially in populous cities (Zhao and Deng, 2012).

Understanding the crash mechanism is a high priority in improving the traffic safety of urban expressways. On urban expressways, vehicles entering from on-ramps and exiting to off-ramps are the major factors that result in traffic interruptions, which play an essential role in many of the vehicle crashes on urban expressways. According to AASHTO (2001), the arrangement of on-ramps and off-ramps has a significant impact on road safety. A few scholars have carried out pioneering studies evaluating the safety effects of highway ramps and acceleration lanes. For example, Bared et al. (1999) examined the impact of acceleration and deceleration lane lengths on traffic safety; Chen et al. (2009) evaluated the safety effects of the number and arrangement of lanes on freeway off-ramps; Liu et al. (2010) discussed three types of lane arrangements on freeway sections with closely spaced ramps and their safety effects; Pulugurtha and Bhatt (2010) analyzed the role of geometric characteristics and traffic on crashes in weaving sections. All the existing studies acknowledge that vehicles entering from on-ramps and leaving to off-ramps significantly change drivers' behavior (e.g. encourage lane changing) and thus result in safety issues.

In this study, field observations are conducted in order to understand the impact of ramps. According to the field survey, we find (1) many drivers change from the shoulder lane to the middle lane before on-ramps in order to avoid having to decelerate and give way to merging vehicles, and (2) the traffic flows cross various traffic lanes and locations are thus unbalanced. Evidently, ramps have an impact on the potential crash risks across locations (before on-ramps, between ramps, and after off-ramps) and lanes (shoulder lane, middle lane, and median lane). Unfortunately, to date, the safety effects of the ramps at various locations and across traffic lanes have not been explored.

In this study, two types of potential crash risks based on time to collision (TTC) are applied to analyse the risk impact of ramps at various locations across traffic lanes. TTC is 
the time that remains until a collision between two vehicles would occur if the collision course and speed difference were maintained (Hayward, 1972). TTC is a well-recognized safety indicator for traffic conflicts on highways (Meng and Qu, 2012a) and similar concepts have been applied to maritime safety analysis (Qu et al., 2011; Li et al., 2012). Minderhoud and Bovy (2001) pointed out that the TTC is inversely related to vehicle crash frequencies in road sections. It is widely accepted as a safety indicator for highways. A TTC threshold value is usually chosen to distinguish relatively safe situations from dangerous scenarios exposed to traffic conflicts (or critical encounters). For example, a car-following scenario is considered to be a traffic conflict or dangerous encounter if its corresponding TTC value is smaller than the predetermined threshold. Note that other well-recognized safety indicators (e.g. time integrated time-to-collision and time exposed time-to-collision) could also be used to estimate the potential crash risks.

The contribution of this paper is three-fold. First, this is the first study to look at the lane distribution of crashes on expressways. The drivers could be better aware of the potential risks when they drive along various traffic lanes. Second, two potential risk indices are proposed to evaluate the safety effects of expressway on-ramps and off-ramps. The two indices could be applied by transport agencies to better design the expressway ramps. Third, the different perspectives of drivers and transport agencies are discussed and analyzed, which could be very useful for helping transport agencies to control potential risks from the viewpoints of not only road managers but also road users.

\section{DATA DESCRIPTION}

\subsection{Site Description}

The field data analysed in this paper were collected from road sections on the Beijing North Ring III expressway, China. The data were collected at seven locations (each location has three lanes: a shoulder lane, middle lane and median lane) for 90 minutes during peak hours (see Figure 1) through a traffic management system (based on processing traffic videos). During the observation periods, no traffic crash occurred. The speed limit was $80 \mathrm{~km} / \mathrm{hour}$. The observed data included the vehicle length, speed, headway, and vehicle type of each vehicle in various traffic lanes across these locations. As can be seen in Figure 1, Locations 3, 5 , and 7 refer to expressway sections following off-ramps (50 meters downstream of the offramps). Locations 1 and 6 are two expressway sections occurring before on-ramps (50 meters upstream of the on-ramps). Locations 2 and 4 are two weaving sections in between two ramps. 


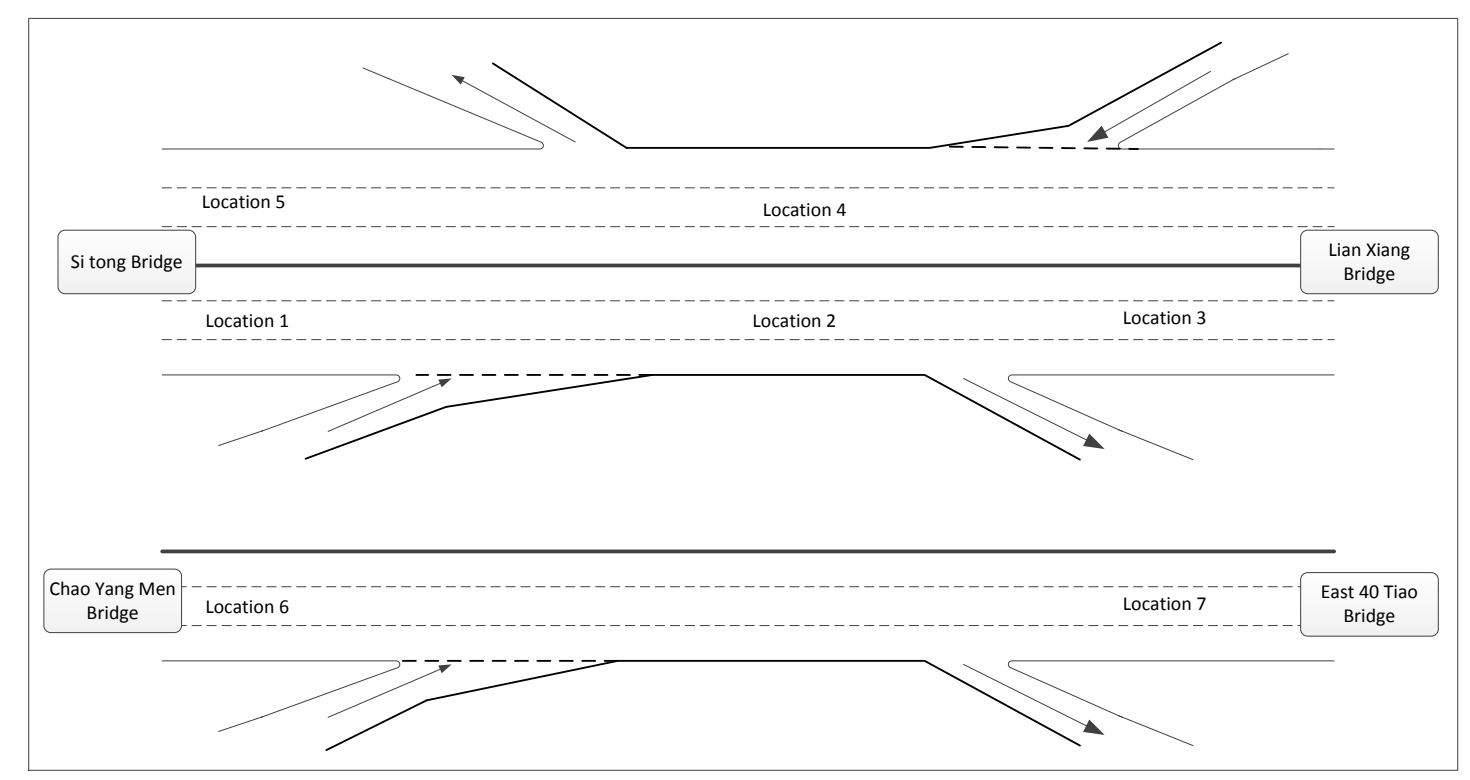

Figure 1: The layout of the survey sites

\subsection{TTC Data Collection}

Figure 2 illustrates a car-following scenario. Let $L_{\text {leader }}$ and $L_{\text {follower }}$ be the positions of the leading and following vehicles at a particular time, respectively; $\dot{L}_{\text {leader }}$ and $\dot{L}_{\text {follower }}$ denote the speeds of the leading and following vehicles at a particular time. According to the TTC definition, namely, the time that remains until a collision between two vehicles would occur if the collision course and speed difference were maintained, the TTC can be mathematically expressed by eq. (1):

$$
T T C=\left\{\begin{array}{cl}
\frac{L_{\text {leader }}-L_{\text {follower }}-l_{\text {leader }}}{\dot{L}_{\text {follower }}-\dot{L}_{\text {leader }}}, & \text { if } \dot{L}_{\text {follower }}>\dot{L}_{\text {leader }} \\
\infty & \text { otherwise }
\end{array}\right.
$$

where $l_{\text {leader }}$ is the length of the leading vehicle. 


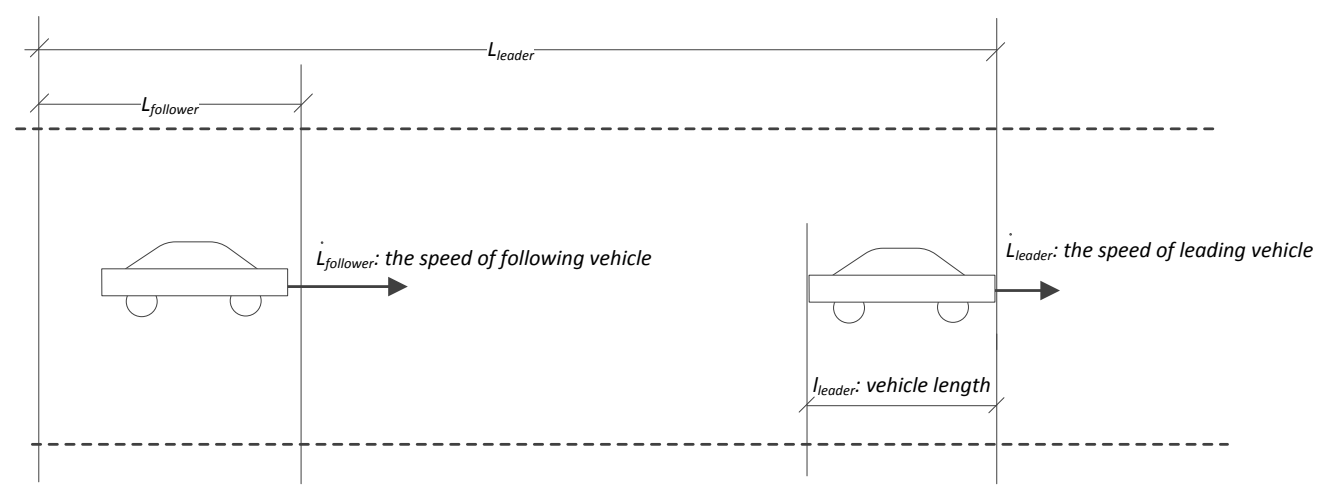

Figure 2: TTC represented by a car-following scenario

The procedure for measuring the TTC with respect to a particular car-following scenario is as follows. First, the length of the leading vehicle $\left(l_{\text {leader }}\right)$, the spot speeds of the two vehicles ( $\dot{L}_{\text {follower }}$ and $\dot{L}_{\text {leader }}$ ), and the time headway ( $h$ ) between the leading and following vehicles can be measured by traffic management systems. Then, according to Vogel (2003), the gap size $\left(L_{\text {leader }}-L_{\text {follower }}-l_{\text {leader }}\right)$ can be estimated by $\left(\dot{L}_{\text {follower }} \times h-l_{\text {leader }}\right)$. Finally, the TTC for this particular car-following scenario can be calculated according to eq. (1). Note that this estimation is based on the assumption that the average speeds can be approximated by the spot speeds.

\section{POTENTIAL CRASH RISKS}

In order to assess the risks on various traffic lanes in different locations, we propose potential crash risks from the perspectives of the transport agencies and individual motorists. Transport agencies tend to evaluate road safety in terms of crash/conflict frequencies for a given road section during a particular time period (Meng et al., 2011; Meng and Qu, 2012b). From their perspective, the risk is interpreted as the overall number of conflicts on a survey road section in a one-hour time period, mathematically,

$$
S R_{i}=\frac{N_{i, T T C<\tau}}{t}
$$

where $N_{i, T T C<\tau}$ denotes the number of TTC samples with a value of $\tau$ or less in road section $i$, and $t$ is the time period of the survey. This risk is termed societal risk in this paper. By contrast, an individual motorist would be more concerned about the risks to which he or she 
is exposed during a journey. In other words, a motorist might interpret the risk as the probability of being involved in a conflict during a journey, mathematically,

$$
I R_{i, j}=\frac{N_{i, T T C<\tau}}{v_{j} \cdot N_{i}}
$$

where $v_{j}$ is the speed of vehicle $j$, and $N_{i}$ is the total number of TTC samples in road section $i$ during the survey period. The risk to an individual motorist is termed the individual risk in this paper. According to eq. (3), the individual risk is estimated by the product of the

probability of a dangerous TTC sample $\left(\frac{N_{i, T T C<\tau}}{N_{i}}\right)$ and the exposure time $\left(\frac{1}{v_{j}}\right)$. The speed has a dual effect on the individual risk. On the one hand, higher speed might result in higher risks in individual car-following scenarios. $N_{i, T T C<\tau}$ thus becomes greater. On the other hand, higher speed implies a shorter journey time for an individual traveler. As a result, the exposure to the risk is for a shorter time. Thus, eq. (3) takes into account not only the risk levels of individual car-following scenarios but also the exposure time. It should be pointed out that the probability of a dangerous TTC sample is calibrated based on the TTC samples under particular conditions.

\section{CRASH RISK IMPACT ANALYSIS}

\subsection{Individual and Societal Crash Risks}

In this study, the locations are categorized into three types: before on-ramps, between onramps and off-ramps, and after off-ramps. For each location, the risks for various traffic lanes (shoulder lane, middle lane, and median lane) are calculated separately according to eqs. (1) and (3). As suggested in Meng and Qu (2012), three seconds is used as the TTC threshold in this study. The results are presented in Table 1.

Table 1: Individual and societal potential crash risks

\begin{tabular}{ccccc}
\hline \multirow{2}{*}{ Location } & Location type & Lane type & Individual risk & Societal risk \\
\hline 1 & 0 & 0 & 0.0019 & 25 \\
& 0 & 0 & 0.0007 & 17 \\
& 0 & 1 & 0.0050 & 26 \\
& 0 & 1 & 0.0004 & 8 \\
& 0 & 2 & 0.0040 & 38 \\
& 0 & 2 & 0.0007 & 13
\end{tabular}




\begin{tabular}{|c|c|c|c|c|}
\hline \multirow[t]{6}{*}{6} & 0 & 0 & 0.0034 & 20 \\
\hline & 0 & 0 & 0.0033 & 18 \\
\hline & 0 & 1 & 0.0031 & 15 \\
\hline & 0 & 1 & 0.0078 & 29 \\
\hline & 0 & 2 & 0.0018 & 9 \\
\hline & 0 & 2 & 0.0052 & 19 \\
\hline \multirow[t]{6}{*}{2} & 1 & 0 & 0.0019 & 25 \\
\hline & 1 & 0 & 0.0008 & 17 \\
\hline & 1 & 1 & 0.0079 & 59 \\
\hline & 1 & 1 & 0.0020 & 22 \\
\hline & 1 & 2 & 0.0122 & 72 \\
\hline & 1 & 2 & 0.0045 & 49 \\
\hline \multirow[t]{6}{*}{4} & 1 & 0 & 0.0007 & 13 \\
\hline & 1 & 0 & 0.0019 & 26 \\
\hline & 1 & 1 & 0.0013 & 18 \\
\hline & 1 & 1 & 0.0042 & 43 \\
\hline & 1 & 2 & 0.0026 & 28 \\
\hline & 1 & 2 & 0.0025 & 23 \\
\hline \multirow[t]{6}{*}{3} & 2 & 0 & 0.0006 & 12 \\
\hline & 2 & 0 & 0.0007 & 16 \\
\hline & 2 & 1 & 0.0017 & 31 \\
\hline & 2 & 1 & 0.0008 & 16 \\
\hline & 2 & 2 & 0.0009 & 11 \\
\hline & 2 & 2 & 0.0017 & 19 \\
\hline \multirow[t]{6}{*}{5} & 2 & 0 & 0.0007 & 13 \\
\hline & 2 & 0 & 0.0018 & 24 \\
\hline & 2 & 1 & 0.0011 & 17 \\
\hline & 2 & 1 & 0.0015 & 17 \\
\hline & 2 & 2 & 0.0007 & 7 \\
\hline & 2 & 2 & 0.0014 & 9 \\
\hline \multirow[t]{6}{*}{7} & 2 & 0 & 0.0015 & 19 \\
\hline & 2 & 0 & 0.0021 & 22 \\
\hline & 2 & 1 & 0.0033 & 44 \\
\hline & 2 & 1 & 0.0033 & 39 \\
\hline & 2 & 2 & 0.0033 & 37 \\
\hline & 2 & 2 & 0.0040 & 41 \\
\hline
\end{tabular}

Notations:

Location types: 0 - before on-ramps; 1 - between on-ramps and off-ramps; 2: after off-ramps. Lane types: 0: median lane; 1 : middle lane; 2 : shoulder lane.

\subsection{Risk Impact Analysis of Location Types}

One-way analysis of variance (ANOVA) in combination of post hoc tests is applied in this paper to analyse the risk impact of location type. The results are presented in Tables 2 and 3. According to the results, we have the following findings: 
(i) The sections between ramps are significantly riskier than those after off-ramps in terms of both individual and societal risk ( $p$ values $=0.029$ and 0.026$)$. The complicated weaving activities between ramps are the leading cause of this difference. After the off-ramps, the individual and societal risks are both reduced because of the reduction in traffic disturbances and traffic volume.

(ii) The individual risk in the sections before on-ramps (Type 0 locations) is very similar to that in the weaving sections (Type 1 locations). However, in terms of societal risk, the sections before on-ramps are significantly safer $(p$ value $=0.04)$ than the weaving sections. Before on-ramps, traffic flows are interrupted by the merging vehicles which results in an individual crash risk similar to that caused by the weaving activities in Type 1 locations. However, higher traffic volume (caused by the merging vehicles) and lower speeds in Type 1 locations result in higher societal risk than in Type 0 locations.

(iii) Type 2 locations (after ramps) are significantly safer than Type 0 in terms of individual risk ( $p$ value $=0.03$ ). However, the two types of location have similar levels of societal risk (19.75 vs. 21.89). Compared to vehicles before on-ramps, those after off-ramps are usually associated with higher speeds. Accordingly, their individual risks are significantly lower thanks to the shorter exposure time. The traffic volume in Type 2 locations is generally higher than that in Type 0 locations. The combined effects cause similar levels of societal risk in both location types.

Table 2: Results of ANOVA for individual crash risk across location types

\begin{tabular}{lll}
\hline & $\mathrm{P}-$ values & Mean values \\
\hline Location 0 vs. 1 & 0.71 (not significant) & 0.0031 vs. 0.0035 \\
Location 0 vs. 2 & 0.03 (significant) & 0.0031 vs. 0.0017 \\
Location 1 vs. 2 & 0.04 (significant) & 0.0035 vs. 0.0017 \\
\hline
\end{tabular}

Table 3: Results of ANOVA for societal crash risk across location types

\begin{tabular}{lll}
\hline & $\mathrm{P}-$ values & Mean values \\
\hline Location 0 vs. 1 & 0.04 (significant) & 19.75 vs. 32.92 \\
Location 0 vs. 2 & 0.59 (not significant) & 19.75 vs. 21.89 \\
Location 1 vs. 2 & 0.05 (significant) & 32.92 vs. 21.89 \\
\hline
\end{tabular}


According to findings (ii) and (iii) above, the safety evaluations in terms of individual and societal risk are not consistent, which is also evidenced by Figure 3. From the persepctive of individual motorists, sections before ramps and after ramps are risky. However, from the perspective of the transport agencies, the sections before and after ramps are relatively safer than the weaving sections.

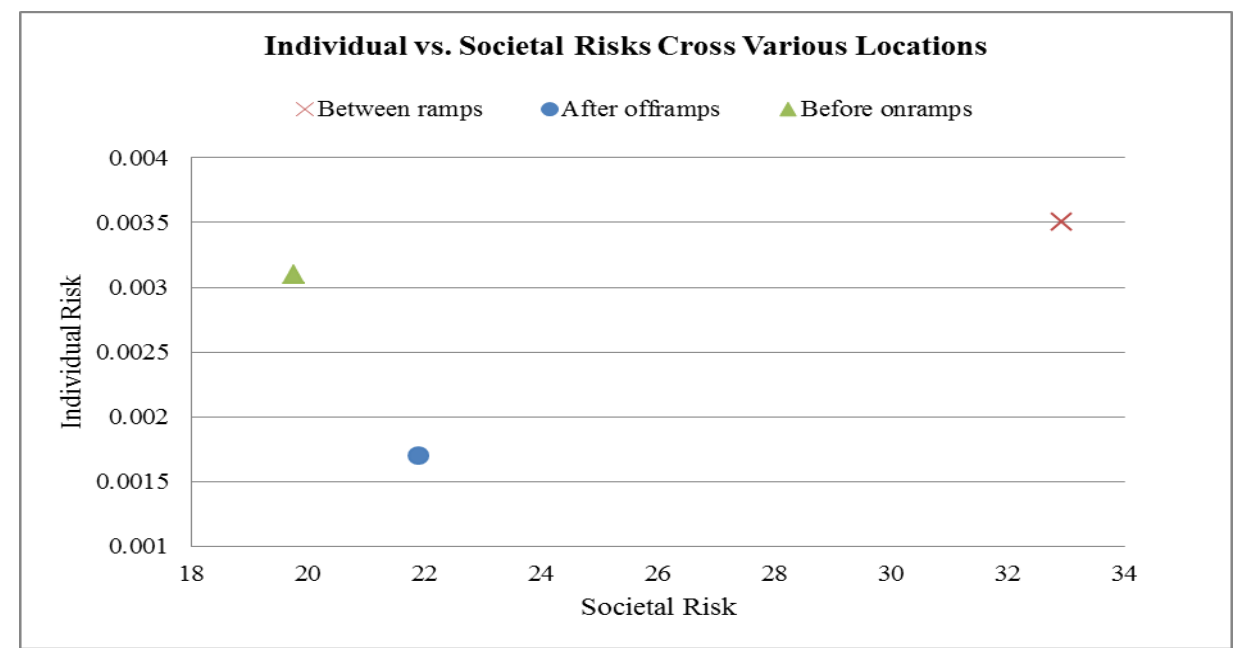

Figure 3: Individual versus societal risk across various locations

\subsection{Risk Impact Analysis of Lane Types}

One-way ANOVA and post hoc test are also applied to examine the risk impact of the lane type. The results are presented in Tables 4 and 5. Some interesting findings are presented below according to the analyses.

(i) There is no significant difference between the shoulder and middle lanes in terms of either individual or societal risk ( $p$ values $=0.92$ and 0.88 ). Vehicles in shoulder lanes are interrupted by merging vehicles and are thus exposed to risky encounters. Some vehicles in the shoulder lane change to the middle lane in order to avoid decelerating and giving way to merging vehicles, which interrupts the middle-lane traffic. The crash risk in the middle lane is thus relatively higher due to the interruptions caused by lane changing.

(ii) The mean societal and individual risks in the median lanes are lower than those of the other two lanes. These differences are significant (at the level of 0.05) or marginally significant (at the level of 0.10 ). This is because vehicles in the median lane are infrequently affected by changing/weaving activities. Therefore, the median lane is comparatively safer than the other two traffic lanes. 
(iii) The individual and societal risk across various traffic lanes show similar patterns $\left(R^{2}=0.97\right)$. According to Figure 4 , the evaluations in terms of the two risk indices are very consistent. The median lanes are relatively safer compared to the other two lanes, from the perspectives of not only the transport agencies but also individual motorists.

Table 4: Results of ANOVA for individual crash risk across traffic lanes

\begin{tabular}{lll}
\hline & $\mathrm{P}-$ values & Mean values \\
\hline Median vs. Middle & 0.04 (significant) & 0.0016 vs. 0.0031 \\
Median vs. Shoulder & 0.06 (marginally significant) & 0.0016 vs. 0.0033 \\
Middle vs. Shoulder & 0.88 (not significant) & 0.0031 vs. 0.0033 \\
\hline
\end{tabular}

Table 5: Results of ANOVA for societal crash risk across traffic lanes

\begin{tabular}{lll}
\hline & $\mathrm{P}-$ values & Mean values \\
\hline Median vs. Middle & 0.05 (significant) & 19.07 vs. 27.43 \\
Median vs. Shoulder & 0.15 (not significant) & 19.07 vs. 26.79 \\
Middle vs. Shoulder & 0.92 (not significant) & 27.43 vs. 26.79 \\
\hline
\end{tabular}

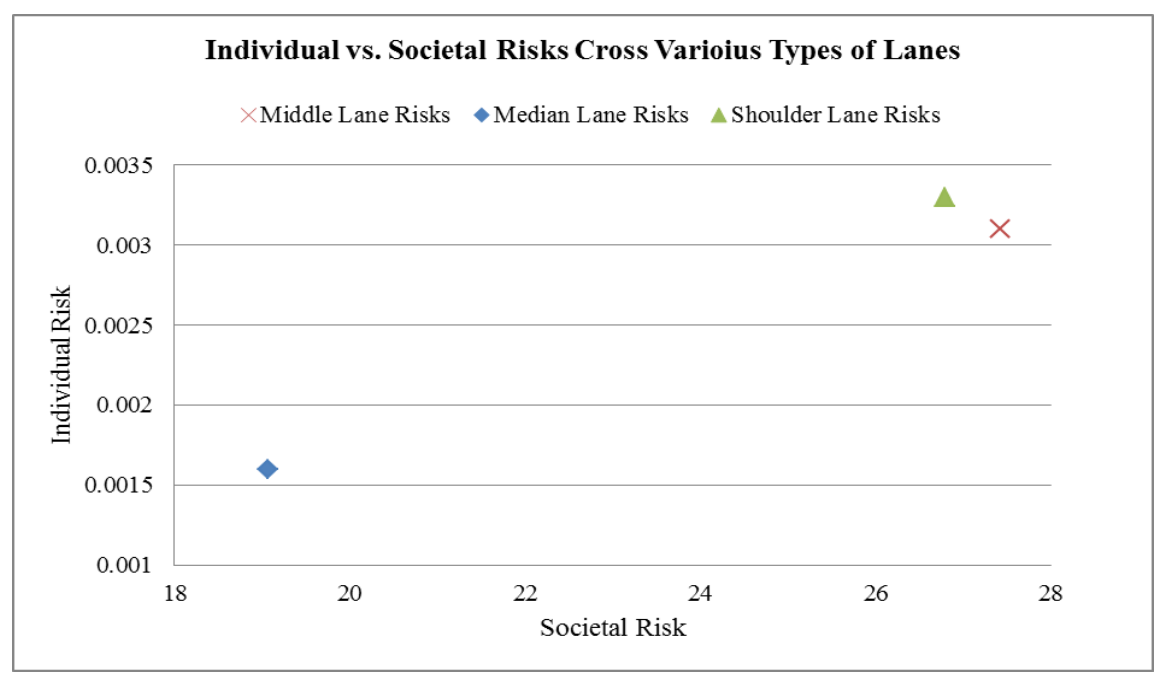

Figure 4: Individual versus societal risk across traffic lanes

\section{CONCLUSIONS}

Traffic interruptions on urban expressways are mainly caused by ramps. Therefore, it is of great importance to analyse the risk impact of ramps in order to cost-effectively implement risk reduction solutions. In this study, we collected traffic trajectory data from 
seven locations on the Beijing Ring III expressway. The locations are categorized into three types: before on-ramps, between ramps, and after off-ramps. Two risk indices based on the notion of TTC, individual risk and societal risk, are proposed to represent the distinct interpretations of risks by transport agencies and individual motorists. One-way ANOVA is applied to analyse the risk impact of ramps on various types of locations across distinct traffic lanes (shoulder lane, middle lane, and median lane). According to the results, median lanes and sections after off-ramps have relatively lower risks than the other lanes and sections. It should be pointed out that the individual and societal risks might not always be consistent since the two risk indices are proposed based on distinct perspectives: transport agencies focus on the crash/conflict frequencies in a road section and individual motorists are more concerned about the probability of being involved in a crash/conflict during their journey.

In this study, we look into the lane distribution of crashes on expressways by using two TTC-based potential risk indices. Road users could be better aware of the potential risks during their journeys when using various traffic lanes. The two indices could be applied by transport agencies to better design expressway ramps. The findings of this research could be very useful for transport agencies aiming to control potential risks from the viewpoints of not only road managers but also road users. In our subsequent research, we will request crash data from transport agencies to establish the relationship between potential crash risk indices and the crash count. The potential for risk-based ramp design on expressways might be another promising research direction.

\section{References}

AASHTO, 2001. A policy on geometric design of highways and streets, Washington, DC.

Bared, J., Giering, L.G., and Warren, L.D., 1999. Safety of evaluation of acceleration and deceleration lane lengths. ITE Journal. 69, 50-54.

BITRE, 2006. Cost of road crashes in Australia 2006. Bureau of Infrastructure, Transport, and Regional Economics. Department of Infrastructure, Transport, Regional Development and Local Government.

Chen, H., Liu, P., Lu, J.J., and Behzadi, B., 2009. Evaluating the safety impacts of the number of arrangement of lanes on freeway exit ramps. Accident Analysis and Prevention. 41, 543-551.

Hayward, J.C., 1972. Near miss determination through use of a scale of danger (traffic records 384). Highway Research Board. Washington, DC. 
Li, S., Meng, Q., and Qu, X., 2012. An overview of maritime waterway quantitative risk assessment models. Risk Analysis, 32, 496-512.

Liu, P., Chen, H, Lu, J.J., and Cao, B., 2010. How lane arrangements on freeway mainlines and ramps affect the safety of freeways with closely spaced entrance and exit ramps. Journal of Transportation Engineering. 136, 614-622.

Meng, Q., and Qu, X., 2012b. Uncertainty propagation in quantitative risk assessment modeling for fire in road tunnels. IEEE Transactions on Systems, Man, and Cybernetics Part C: Applications and Reviews, 42, 1454-1464.

Meng, Q., and Qu, X., 2012a. Estimation of rear-end vehicle crash frequencies in urban road tunnels. Accident Analysis and Prevention. 48, 254-263.

Meng, Q., Qu, X., Yong, K.T., and Wong, Y.H., 2011. QRA model-based risk impact analysis of traffic flow in urban road tunnels. Risk Analysis. 31, 1872-1882.

Minderhoud, M.M., and Bovy, P.H.L., 2001. Extended time-to-collision measures for road traffic safety assessment. Accident Analysis and Prevention. 33, 89-97.

Pei, X., Wong, S.C., and Sze, N.N., 2012. The roles of exposure and speed in road safety analysis. Accident Analysis and Prevention 48, 464-471.

Pulugurtha, S.S., and Bhatt, J., 2010. Evaluating the role of weaving section characteristics and traffic on crashes in weaving sections. Traffic Injury Prevention. 11, 104-113.

Qu, X., Kuang, Y., Oh, E., and Jin, S., 2014. Safety evaluation for expressways: A comparative study for macroscopic and microscopic indicators. Traffic Injury Prevention. $15,89-93$.

Qu, X., Meng, Q., and Suyi, L., 2011. Ship collision risk assessment for the Singapore Strait. Accident Analysis and Prevention, 43, 2030-2036.

Vogel, K., 2003. A comparison of headway and time to collision as safety indicators. Accident Analysis and Prevention. 35, 427-433.

Zhao, J., and Deng, W., 2012. Traffic accidents on expressways: New threat to China. Traffic Injury Prevention. 13, 230-238. 